\title{
Association between anion gap and mortality of aortic aneurysm in intensive care unit after open surgery
}

\author{
Yijing Gao ${ }^{3 \dagger}$, Zilin Hong ${ }^{2 \dagger}$, Runnan Shen ${ }^{2 \dagger}$, Shiran Zhang ${ }^{2}$, Guochang You², Jie Chen², Xushun Guo², \\ Senyi Peng ${ }^{2}$ and Kai Huang ${ }^{1,2^{*}}$
}

\begin{abstract}
Background: There has not been a well-accepted prognostic model to predict the mortality of aortic aneurysm patients in intensive care unit after open surgery repair. Otherwise, our previous study found that anion gap was a prognosis factor for aortic aneurysm patients. Therefore, we wanted to investigate the relationship between anion gap and mortality of aortic aneurysm patients in intensive care unit after open surgery repair.
\end{abstract}

Methods: From Medical Information Mart for Intensive Care III, data of aortic aneurysm patients in intensive care unit after open surgery were enrolled. The primary clinical outcome was defined as death in intensive care unit. Univariate analysis was conducted to compare the baseline data in different groups stratified by clinical outcome or by anion gap level. Restricted cubic spline was drawn to find out the association between anion gap level and mortality. Subgroup analysis was then conducted to show the association in different level and was presented as frost plot. Multivariate regression models were built based on anion gap and were adjusted by admission information, severity score, complication, operation and laboratory indicators. Receiver operating characteristic curves were drawn to compare the prognosis ability of anion gap and simplified acute physiology score II. Decision curve analysis was finally conducted to indicate the net benefit of the models.

Results: A total of 405 aortic aneurysm patients were enrolled in this study and the in-intensive-care-unit (inICU) mortality was 6.9\%. Univariate analysis showed that elevated anion gap was associated with high mortality ( $P$ value $<0.001$ ), and restricted cubic spline analysis showed the positive correlation between anion gap and mortality. Receiver operating characteristic curve showed that the mortality predictive ability of anion gap approached that of simplified acute physiology score II and even performed better in predicting in-hospital mortality $(P$ value $<0.05)$. Moreover, models based on anion gap showed that $1 \mathrm{mEq} / \mathrm{L}$ increase of anion gap improved up to $42.3 \%$ (95\% confidence interval 28.5-59.8\%) risk of death.

Conclusions: The level of serum anion gap was an important prognosis factor for aortic aneurysm mortality in intensive care unit after open surgery.

Keywords: Aortic aneurysm, Anion gap, Prognosis, Open surgery, Intensive care unit

*Correspondence: huangk37@mail.sysu.edu.cn

${ }^{\dagger}$ Yijing Gao, Zilin Hong and Runnan Shen contributed equally to this work

1 Department of Cardiovascular Surgery, Sun Yat-Sen Memorial

Hospital, Sun Yat-Sen University, No. 33, Yingfeng Road, Haizhu District,

Guangzhou 510000, Guangdong Province, China

Full list of author information is available at the end of the article

\section{Background}

Aneurysm is the second most frequent disease of the aorta after atherosclerosis [1]. Aortic aneurysm (AA) accounts for over 10,000 deaths in America annually [2]. AA is subdivided into thoracic aortic aneurysm (TAA) 
and abdominal aortic aneurysm (AAA). Despite diagnostic advances, in-time diagnosis of AAs in an early stage is difficult due to lack of classic symptoms. AAAs are found in up to $8 \%$ of men aged 65 years, yet usually remain asymptomatic until they rupture [3]. Early TAAs remain asymptomatic as well, until aortic dissection or rupture occurs, which is related to a high mortality of approximately $50 \%$ [4]. With the aging of population, the incidence of aortic diseases will increase further [5].

Nowadays, surgery is still the main treatment of AA, of which open surgery repair (OSR) and endovascular aortic repair (EVAR) are the most common two. In the recent years, the latter is used more often because of its low mortality and probability of comorbidities in perioperative phase, since EVAR avoids ischemia of organ and large trauma, compared with OSR [1]. However, OSR is still an important choice of AA patients due to its efficacy within a wide range. First of all, OSR shows long-term advantages, compared with EVAR. The Dutch Randomized Endovascular Aneurysm Management (DREAM) trial shows that although EVAR is better than OSR in aspect of 30-day mortality, comorbidity and length of stay (LOS) in hospital, there is no significant difference in 6-year follow-up after surgery, and reintervention rate of EVAR is even higher than that of OSR [6]. What's more, EVAR1, a clinical trial reports that patients who have received EVAR have a lower survival rate than patients that have received OSR in 15-year follow-up, mainly owing to an increase of secondary aneurysmal sac rupture in EVAR group [6]. Therefore, OSR is still a treatment worthy of consideration. Moreover, OSR is still the standard when dealing with some types of AA. In cases of Marfan disease and other connective tissue diseases, when dealing with TAA in the descending part of the aorta, OSR should be preferred over thoracic endovascular aortic repair (TEVAR), since there is no evidence supporting any use of TEVAR in patients with connective tissue disease, except in emergency situations in order to get initial stabilization as a bridge to definitive surgical therapy [1]. As for AAA, in patients with complex aortic anatomy, including those with aneurysms in close proximity to or involving the renal arteries, EVAR is unsuitable, and OSR remains the standard [1]. In addition, OSR is a remedy when EVAR fails or causes comorbidities. For instance, OSR is a selection of prompt treatment to secondary endoleak following EVAR and TEVAR [7]. Since OSR has high efficacy but still with problems in safety simultaneously, the prognosis of it is still worthy of enough consideration. However, it seems that the existing predictive models for AA mortality have several shortcomings, and are not useful and practical for clinical decision making [8]. Thus, new predictors of AA prognosis should be discovered. What's more, elevated anion gap (AG) is a risk factor for mortality of critically ill patients. Kim et al indicates that corrected AG at ICU admission may be used to predict mortality in children, regardless of underlying etiology [9]. Since there had not been a research that study the relationship between AG and mortality of AA, in our previous study, we conducted a research aiming to confirm AG as a prognosis factor for prognosis of AA patients in ICU, using the patient information obtained from Medical Information Mart for Intensive Care III (MIMIC-III), and found admission serum anion gap might serve as a strong predictor of ICU mortality for AA patients [10]. However, in that study we did not include patients with thoracoabdominal aneurysm, and we did not focus on OSR specially, which has higher perioperative mortality. Since OSR has certain risk of adverse prognosis, especially in perioperative phase, it's necessary to build and find reliable prognosis factors of OSR. By conducting the current research, we aimed to find out that whether AG had a better prognosis effect when dealing with the subgroup receiving OSR. Moreover, we wanted to use more kinds of statistical tools to find out the association between AG and clinical outcome and estimate the clinical net benefit of the predictive models.

\section{Methods \\ Data retrieval}

The data used in this study were all obtained from MIMIC-III, a free database that integrates in-hospital data of over 50,000 ICU patients in Beth Israel Deaconess Medical Center in Boston, Massachusetts from 2001 to 2012 [11]. In MIMIC-III, the diagnoses of a patient are defined by International Classification of Diseases, Clinical Modification (ICD-9-CM) [11]. Through International Classification of Diseases 9 (ICD-9) code, first hospital admission and first ICU admission of AA patients were included. Exclusion standards were as follows: (1) not first hospital and ICU admission; (2) age < 18; (3) ICU stay<24 h; (4) without an AG record in first day after admission; (5) did not receive open surgery.

The baseline data of patients that we obtained included general condition, comorbidity, laboratory indicators on admission, treatment and severity scores. Comorbidity were defined by ICD-9 code as well on same hospital admission. Among laboratory indicators, AG, creatinine, blood urea nitrogen, partial thromboplastin time (PTT), international normalized ratio (INR), prothrombin time (PT) and white blood cell count were maximum value detected in first day of ICU admission, while bicarbonate, hematocrit, hemoglobin and platelet count were the minimum value. For missing value, we performed single imputation for the whole dataset based on the complete conditional specification and used predictive mean matching method to full-fill them [12]. A sensitivity 
analysis was also conducted to access the difference between original and imputed data. For those who are interested in the details of this sensitivity analysis, check Additional file 1 for more information. The data obtained from MIMIC-III were stored in PostgreSQL (Version: 10.12). Creation of materialized view and extraction of relevant data were conducted in PostgreSQL (Version: 10.12) through mimic-code for MIMIC-III $[13,14]$.

\section{Clinical outcome}

The primary outcome of patients was death in ICU. The secondary outcome included death in hospital, death within 90 days and death within 365 days.

\section{Data analysis}

Baseline data of AA patients in ICU after OSR were grouped by clinical outcomes. The continuous data's normality was tested through Shapiro-Wilk normality test. And in two independent groups comparison, continuous variables with normal distribution would be represented by mean with standard deviation (SD), compared through t-test. Continuous variables with abnormal distribution would be represented by median and interquartile range, compared through Wilcoxon rank sum test. Categorical variables would be represented by frequency and percentage, compared through chi-square test. To find out whether AG had a predictive effect of mortality, we stratified the baseline data by AG level $(<12 \mathrm{mEq} / \mathrm{L}$, $12-16 \mathrm{mEq} / \mathrm{L},>16 \mathrm{mEq} / \mathrm{L}$ ) on ICU admission. The continuous data's normality was tested with Shapiro-Wilk normality test. In more than two groups comparison, continuous variables with normal distribution would be represented by mean with $\mathrm{SD}$, compared through oneway ANOVA test. Continuous variables with abnormal distribution would be represented by median and interquartile range, compared through Kruskal-Wallis rank sum test. Categorical variables would be represented by frequency and percentage, compared through chi-square test. To illuminate the effects of AG on risk of ICU mortality, we drew 4 restricted cubic splines (RCSs) which were adjusted for different kinds of variables or nonadjusted. The first one model was crude and not adjusted; The second model was adjusted for admission information and severity score, including admission type, age, gender, aortic rupture, Sequential Organ Failure Assessment (SOFA), Simplified Acute Physiology Score II (SAPSII) and Glasgow Coma Scale (GCS); The third model was adjusted for complication and operation, including sepsis, chronic pulmonary diseases, peripheral vascular diseases, hypertension, renal failure, coagulopathy, fluid and electrolyte disorders, extracorporeal circulation, bypass surgery, ventilation on first day and urine output on first day; The fourth model was adjusted for laboratory indicators, including bicarbonate, creatinine, blood urea nitrogen, hematocrit, hemoglobin, PTT, PT, INR, white blood cell count and platelet count.

Logistic regression was conducted to show subgroup analysis of association between serum AG and ICU mortality and was adjusted for SOFA score. Different groups were stratified by type of admission, rupture, age, gender, sepsis, chronic pulmonary diseases, renal failure, coagulopathy diseases and fluid electrolyte disorders. Finally, the result of the subgroup analysis was presented as frost plot.

We conducted some analyses to compare the risk discrimination of AG and SAPSII in aspect of different clinical outcomes, including ICU-mortality, hospitalmortality, 90-day mortality, and 1-year mortality. Area under curve (AUC) of continuous AG and SAPSII were calculated respectively and compared by Delong's test of their correlated receiver operating characteristic (ROC) curves [15]. Net reclassification index (NRI) and integrated discrimination improvement (IDI) between AG model and SAPSII model were also calculated and tested [16].

To assess the effects of AG on ICU mortality, models including AG were analyzed through multivariable logistic regression. Among these models, one was a crude model which only includes AG gap, while the other 4 models were adjusted for different variable groups. Model I was adjusted for admission information and severity score, including admission type, age, gender, aortic rupture, SOFA, SAPSII and GCS; Model II was adjusted for complication and operation, including sepsis, chronic pulmonary diseases, peripheral vascular diseases, hypertension, renal failure, coagulopathy, fluid and electrolyte disorders, extracorporeal circulation, bypass surgery, ventilation on first day and urine output on first day; Model III was adjusted for laboratory indicators, including bicarbonate, creatinine, blood urea nitrogen, hematocrit, hemoglobin, PTT, PT, INR, white blood cell count and platelet count; Model IV was adjusted for admission information, severity score, complication, operation and laboratory indicators above. Moreover, decision curve analysis (DCA) was drawn to show the net benefit and clinical usefulness of different models adjusted for different kinds of variables [17].

The data analysis above were all finished in R software (Version: 3.6.1).

\section{Results \\ Enrollment}

The process of enrollment is shown in Fig. 1. Firstly, through inclusion of patients with ICD-9 code 44114414, 4416, 4417 (4411: thoracic aneurysm, ruptured; 


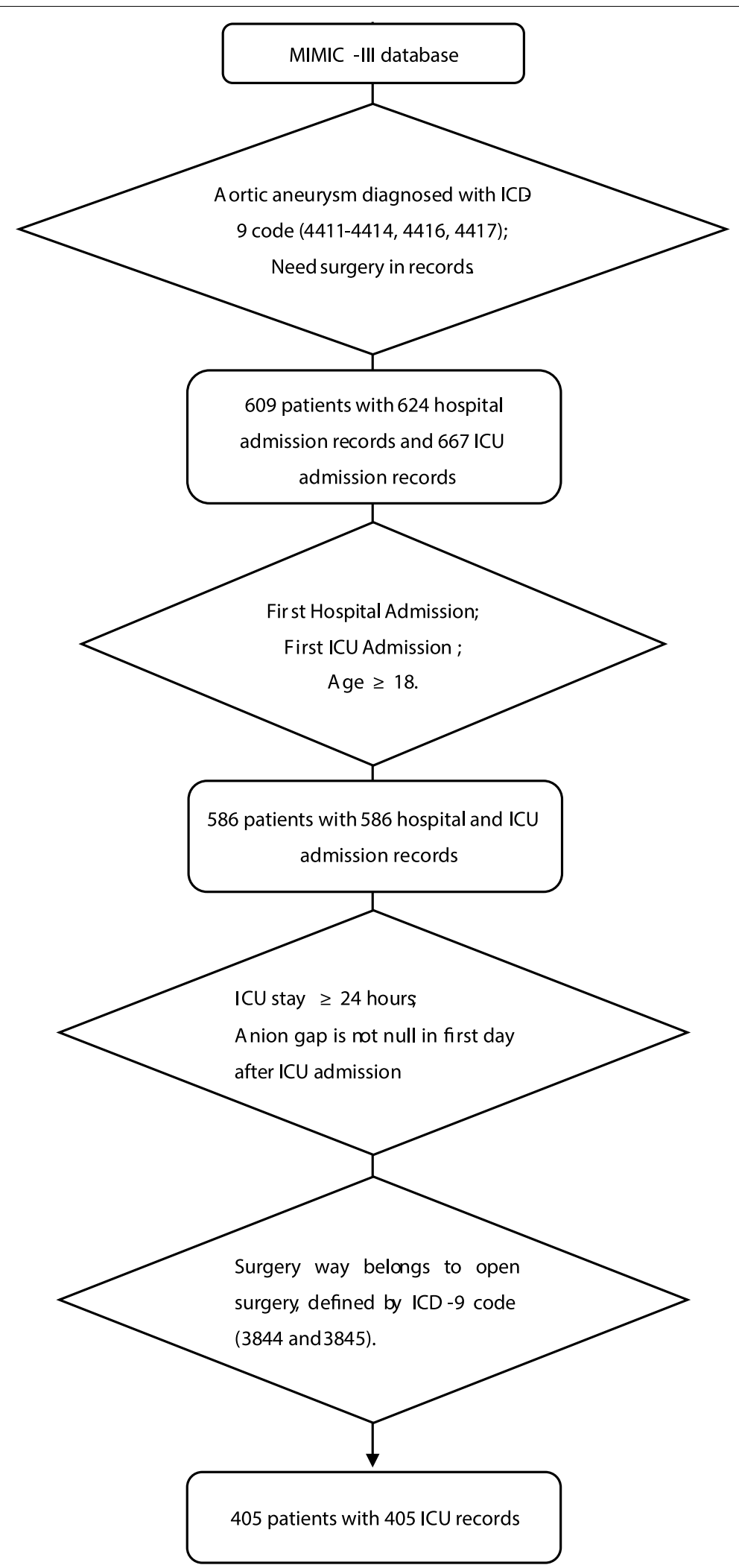

Fig. 1 Flow chart of the study population. In-ICU AA patients with open surgery records were enrolled. Abbreviation: MIMIC-III, Medical Information Mart for Intensive Care III; ICD-9, international Classification of Diseases, 9th Revision; ICU, intensive care unit 
Table 1 Baseline data of aortic aneurysm patients in intensive care unit after open surgery

\begin{tabular}{|c|c|c|c|c|}
\hline Variables & & $\begin{array}{l}\text { ICU-Survival } \\
(\mathrm{N}=377)\end{array}$ & $\begin{array}{l}\text { ICU-Death } \\
(\mathrm{N}=28)\end{array}$ & $P$ value \\
\hline \multicolumn{5}{|c|}{ General condition } \\
\hline & Admission type & & & $<0.001$ \\
\hline & ELECTIVE & $282(74.8 \%)$ & $8(28.6 \%)$ & \\
\hline & EMERGENCY & $86(22.8 \%)$ & $20(71.4 \%)$ & \\
\hline & URGENT & $9(2.4 \%)$ & $0(0.0 \%)$ & \\
\hline & Death in hospital & $2(0.5 \%)$ & $28(100.0 \%)$ & $<0.001$ \\
\hline & Death within 90 days & $8(2.1 \%)$ & $28(100.0 \%)$ & $<0.001$ \\
\hline & Death within 365 days & $20(5.3 \%)$ & $28(100.0 \%)$ & $<0.001$ \\
\hline & LOS in hospital (days) & $9.06[6.27,14.15]$ & $12.00[4.07,19.01]$ & $<0.001$ \\
\hline & LOS in intensive care unit (days) & $3.08[1.86,6.81]$ & $11.88[2.65,19.19]$ & 0.782 \\
\hline & Age (years) & $68.58[59.14,75.40]$ & $76.80[70.14,80.96]$ & $<0.001$ \\
\hline & Type of aortic aneurysm & & & $<0.001$ \\
\hline & Abdominal aneurysm without mention of rupture & $101(26.8 \%)$ & $8(28.6 \%)$ & \\
\hline & Abdominal aneurysm, ruptured & $25(6.6 \%)$ & $10(35.7 \%)$ & \\
\hline & Thoracic aneurysm without mention of rupture & $216(57.3 \%)$ & $5(17.9 \%)$ & \\
\hline & Thoracic aneurysm, ruptured & $5(1.3 \%)$ & $1(3.6 \%)$ & \\
\hline & Thoracoabdominal aneurysm, ruptured & $5(1.3 \%)$ & $2(7.1 \%)$ & \\
\hline & Thoracoabdominal aneurysm, without mention of rupture & $25(6.6 \%)$ & $2(7.1 \%)$ & \\
\hline & Male & $248(65.8 \%)$ & $13(46.4 \%)$ & 0.063 \\
\hline & Aortic rupture & $35(9.3 \%)$ & $13(46.4 \%)$ & $<0.001$ \\
\hline \multicolumn{5}{|c|}{ Comorbidity } \\
\hline & Sepsis & $6(1.6 \%)$ & $6(21.4 \%)$ & $<0.001$ \\
\hline & Chronic pulmonary diseases & $81(21.5 \%)$ & $7(25.0 \%)$ & 0.843 \\
\hline & Peripheral vascular diseases & $198(52.5 \%)$ & $9(32.1 \%)$ & 0.059 \\
\hline & hypertension & $22(5.8 \%)$ & $3(10.7 \%)$ & 0.53 \\
\hline & Renal failure & $27(7.2 \%)$ & $4(14.3 \%)$ & 0.318 \\
\hline & Coagulopathy & $66(17.5 \%)$ & $11(39.3 \%)$ & 0.01 \\
\hline & Fluid and electrolyte disorders & $82(21.8 \%)$ & $7(25.0 \%)$ & 0.87 \\
\hline \multicolumn{5}{|c|}{ Laboratory indicators on admission } \\
\hline & Anion gap (mEq/L) & $12.00[11.00,14.00]$ & $17.00[15.75,22.00]$ & $<0.001$ \\
\hline & Bicarbonate (mEq/L) & $23.00[21.00,25.00]$ & $17.50[15.75,21.00]$ & $<0.001$ \\
\hline & Creatinine $(\mathrm{mg} / \mathrm{dL})$ & $1.00[0.80,1.20]$ & $1.65[1.15,2.10]$ & $<0.001$ \\
\hline & Blood urea nitrogen (mg/dL) & $17.00[13.00,21.00]$ & $27.00[20.75,34.25]$ & $<0.001$ \\
\hline & Hematocrit (\%) & $25.00[21.10,29.00]$ & $24.65[21.00,26.10]$ & 0.266 \\
\hline & Hemoglobin (g/dL) & $8.50[7.40,9.80]$ & $8.35[7.00,8.80]$ & 0.106 \\
\hline & PTT (sec) & $41.45[34.35,51.55]$ & $76.45[43.55,122.25]$ & $<0.001$ \\
\hline & PT (sec) & $15.80[14.60,17.50]$ & $17.55[14.75,19.40]$ & 0.074 \\
\hline & INR & $1.50[1.30,1.70]$ & $1.80[1.37,2.32]$ & 0.026 \\
\hline & $\begin{array}{l}\text { white blood cell count } \\
(\mathrm{K} / \mu \mathrm{L})\end{array}$ & $12.50[9.90,15.70]$ & $14.65[10.38,16.95]$ & 0.048 \\
\hline & Platelet count $(\mathrm{K} / \mu \mathrm{L})$ & $124.00[89.75,156.00]$ & $84.50[61.00,120.00]$ & $<0.001$ \\
\hline \multicolumn{5}{|l|}{ Treatment } \\
\hline & Extracorporeal circulation & $243(64.5 \%)$ & $7(25.0 \%)$ & $<0.001$ \\
\hline & Bypass surgery & $14(3.7 \%)$ & $2(7.1 \%)$ & 0.692 \\
\hline & Ventilation in first day & $356(94.4 \%)$ & $26(92.9 \%)$ & 1 \\
\hline & Urine output on first day (ml) & $2026.00[1393.50,3038.75]$ & $948.00[344.00,1428.00]$ & $<0.001$ \\
\hline \multicolumn{5}{|c|}{ Severity scores } \\
\hline & GCS & $15.00[14.25,15.00]$ & $15.00[15.00,15.00]$ & 0.133 \\
\hline & SOFA & $5.00[4.00,8.00]$ & $9.00[7.75,11.25]$ & $<0.001$ \\
\hline
\end{tabular}


Table 1 (continued)

\begin{tabular}{cllll}
\hline Variables & & $\begin{array}{l}\text { ICU-Survival } \\
(\mathbf{N}=\mathbf{3 7 7})\end{array}$ & $\begin{array}{l}\text { ICU-Death } \\
(\mathbf{N}=\mathbf{2 8})\end{array}$ \\
\hline SAPSII & $32.00[26.00,42.00]$ & $49.50[43.75,65.00]$
\end{tabular}

The continuous data's normality was tested with Shapiro-Wilk normality test. And in two independent groups compare, continuous variables with normal distribution would be represented by mean with standard deviation (SD), compared with t-test. Continuous variables with abnormal distribution would be represented by median and interquartile range, compared with Wilcoxon rank sum test. Categorical variables would be represented by frequency and percentage, compared with chi-square test. $0.2 \%$ of patients had unknown value for hemoglobin; $0.2 \%$ for platelet; $2.2 \%$ for PTT; $2.2 \%$ for PT; $2.2 \%$ for INR; $0.5 \%$ for white blood cell count; $0.4 \%$ for urine output on first day; $0.7 \%$ for GCS score. Abbreviation: LOS, length of stay; PTT, partial thromboplastin time; PT, prothrombin time; INR, international normalized ratio; GCS, Glasgow Coma Scale; SOFA, sequential organ failure assessment; SAPSII, simplified acute physiology score II

4412: thoracic aneurysm without mention of rupture; 4413: abdominal aneurysm, ruptured; 4414: abdominal aneurysm without mention of rupture; 4416: thoracoabdominal aneurysm, ruptured; 4417: thoracoabdominal aneurysm, without mention of rupture) and exclusion of patients without surgery in records, 609 AA patients with 624 hospital admission records and 667 ICU admission records were obtained from MIMIC-III. Secondly, among these patients and records, first hospital admission and first ICU admission record of patients no less than 18 years old were included, while other records were excluded. Through this we obtained 586 patients with 586 hospital and ICU admission records. Thirdly, by using ICD-9 code 3844 (resection of vessel with replacement, aorta, abdominal) and 3845 (Resection of vessel with replacement, thoracic vessels), we enrolled the patients whose surgery way belongs to open surgery, and 405 patients with 405 ICU records were enrolled in the study eventually. Among them, 144 were AAA patients, 227 were TAA patients, 34 were thoracoabdominal aortic aneurysm patients.

\section{Baseline data}

As is shown in Table 1, among these 405 patients, 377 (93.1\%) survived and $28(6.9 \%)$ died in ICU. Sensitivity analysis showed no difference between original and imputed data, which is shown in Stable 1. Comparing the ICU survival group and the death group, in general condition, admission type, death in hospital, death within 90 days, death within 365 days, LOS in hospital, age, type of AA and aortic rupture were statistically significant $(P$ value $<0.05)$; in comorbidity, sepsis and coagulopathy were statistically significant $(P$ value $<0.05)$; in laboratory indicators on admission, AG, bicarbonate, creatinine, blood urea nitrogen, PTT, INR, white blood cell count and platelet count were statistically significant $(P$ value $<0.05)$; in treatment, extracorporeal circulation and urine output on first day were statistically significant $(P$ value $<0.05)$; in severity scores, SOFA and SAPSII were statistically significant $(P$ value $<0.05)$.

\section{Risk stratification}

As is shown in Table 2, comparing the baseline data of 3 groups whose AG levels were respectively $<12 \mathrm{mEq} / \mathrm{L}$, between $12-16 \mathrm{mEq} / \mathrm{L}$, and $>16 \mathrm{mEq} / \mathrm{L}$, all factors of general condition, including admission type, death in ICU, death in hospital, death within 90 days, death within 365 days, LOS in hospital, LOS in ICU, type of AA, age, gender, aortic rupture, were statistically significant $(P$ value $<0.05)$; in comorbidity, sepsis, peripheral vascular diseases and coagulopathy were statistically significant ( $P$ value $<0.05)$; in laboratory indicators on admission, AG, bicarbonate, creatinine, blood urea nitrogen, PTT, prothrombin time (PT), INR and platelet count were statistically significant $(P$ value $<0.05)$; in treatment, extracorporeal circulation used and urine output in first day were statistically significant $(P$ value $<0.05)$; in severity scores, SOFA and SAPSII were statistically significant $(P$ value $<0.05)$.

As is shown in Table 3, comparing prediction abilities of AG and SAPSII model, in predicting in-hospital mortality, AG model had a higher AUC than SAPSII model (0.861 versus 0.833 ), NRI of which was 0.373 (95\% confidence interval (CI) $0.009-0.738)$ ( $P$ value $<0.05$ ); in predicting 1-year mortality, AG model had a lower AUC than SAPSII model (0.775 versus 0.780$)$, NRI of which was 0.304 (95\% CI $0.007-0.601)(P$ value $<0.05)$ and IDI of which was $0.095(95 \%$ CI $0.022-0.167)(P$ value $<0.05)$. It indicated that AG model was more capable of predicting postoperative mortality than SAPSII model, although SAPSII model performed better in predicting 1-year mortality.

\section{Relationship between AG and mortality}

As is shown in Fig. 2, RCS analysis indicated that the level of AG had a statistically significant relationship with ICU mortality $\left(P_{\text {total }}\right.$ value $\left.<0.001\right)$. As the level of AG increased, the risk of ICU death increased at the same time. It could be seen that the growing tendency of mortality became sharper when the level of AG is above 
Table 2 Baseline data of studying aortic aneurysm stratified by anion gap on ICU admission

\begin{tabular}{|c|c|c|c|c|c|}
\hline Variables & & $\begin{array}{l}\text { Anion gap }<12 \mathrm{mEq} / \mathrm{L} \\
(\mathrm{N}=151)\end{array}$ & $\begin{array}{l}\text { Anion gap between } \\
12-16 \mathrm{mEq} / \mathrm{L} \\
(\mathrm{N}=200)\end{array}$ & $\begin{array}{l}\text { Anion gap }>16 \mathrm{~m} \mathrm{Eq} / \mathrm{L} \\
(\mathrm{N}=54)\end{array}$ & $P$ value \\
\hline \multicolumn{6}{|c|}{ General condition } \\
\hline & Admission type & & & & $<0.001$ \\
\hline & ELECTIVE & $121(80.1 \%)$ & $153(76.5 \%)$ & $16(29.6 \%)$ & \\
\hline & EMERGENCY & $27(17.9 \%)$ & $42(21.0 \%)$ & $37(68.5 \%)$ & \\
\hline & URGENT & $3(2.0 \%)$ & $5(2.5 \%)$ & $1(1.9 \%)$ & \\
\hline & Death in ICU & $1(0.7 \%)$ & $8(4.0 \%)$ & $19(35.2 \%)$ & $<0.001$ \\
\hline & Death in hospital & $2(1.3 \%)$ & $8(4.0 \%)$ & $20(37.0 \%)$ & $<0.001$ \\
\hline & Death within 90 days & $4(2.6 \%)$ & $11(5.5 \%)$ & $21(38.9 \%)$ & $<0.001$ \\
\hline & Death within 365 days & $6(4.0 \%)$ & $15(7.5 \%)$ & $27(50.0 \%)$ & $<0.001$ \\
\hline & LOS in hospital (days) & $7.36[6.12,11.01]$ & $9.24[6.27,16.87]$ & $13.52[9.26,21.77]$ & $<0.001$ \\
\hline & LOS in ICU (days, ) & $2.25[1.33,3.98]$ & $3.39[2.01,9.18]$ & $8.95[3.01,20.01]$ & $<0.001$ \\
\hline & Type of aortic aneurysm & & & & $<0.001$ \\
\hline & $\begin{array}{l}\text { Abdominal aneurysm without mention of } \\
\text { rupture }\end{array}$ & $34(22.5 \%)$ & $63(31.5 \%)$ & $12(22.2 \%)$ & \\
\hline & Abdominal aneurysm, ruptured & $2(1.3 \%)$ & $12(6.0 \%)$ & $21(38.9 \%)$ & \\
\hline & $\begin{array}{l}\text { Thoracic aneurysm without mention of } \\
\text { rupture }\end{array}$ & $105(69.5 \%)$ & $109(54.5 \%)$ & $7(13.0 \%)$ & \\
\hline & Thoracic aneurysm, ruptured & $1(0.7 \%)$ & $1(0.5 \%)$ & $4(7.4 \%)$ & \\
\hline & Thoracoabdominal aneurysm, ruptured & $2(1.3 \%)$ & $2(1.0 \%)$ & $3(5.6 \%)$ & \\
\hline & $\begin{array}{l}\text { Thoracoabdominal aneurysm, without men- } \\
\text { tion of rupture }\end{array}$ & $7(4.6 \%)$ & $13(6.5 \%)$ & $7(13.0 \%)$ & \\
\hline & Age (years) & $64.04[56.20,73.00]$ & $70.35[60.43,76.72]$ & $73.06[69.39,79.86]$ & $<0.001$ \\
\hline & Male & $95(62.9 \%)$ & $140(70.0 \%)$ & $26(48.1 \%)$ & 0.011 \\
\hline & Aortic rupture & $5(3.3 \%)$ & $15(7.5 \%)$ & $28(51.9 \%)$ & $<0.001$ \\
\hline \multicolumn{6}{|c|}{ Comorbidity } \\
\hline & Sepsis & $2(1.3 \%)$ & $4(2.0 \%)$ & $6(11.1 \%)$ & 0.001 \\
\hline & Chronic pulmonary diseases & $31(20.5 \%)$ & $48(24.0 \%)$ & $9(16.7 \%)$ & 0.461 \\
\hline & Peripheral vascular diseases & $90(59.6 \%)$ & 99 (49.5\%) & $18(33.3 \%)$ & 0.003 \\
\hline & hypertension & $7(4.6 \%)$ & $13(6.5 \%)$ & $5(9.3 \%)$ & 0.463 \\
\hline & Renal failure & $7(4.6 \%)$ & $17(8.5 \%)$ & $7(13.0 \%)$ & 0.116 \\
\hline & Coagulopathy & $19(12.6 \%)$ & $35(17.5 \%)$ & $23(42.6 \%)$ & $<0.001$ \\
\hline & Fluid and electrolyte disorders & $29(19.2 \%)$ & $43(21.5 \%)$ & $17(31.5 \%)$ & 0.17 \\
\hline \multicolumn{6}{|c|}{ Laboratory indicators on admission } \\
\hline & Anion gap (mEq/L) & $10.00[9.00,11.00]$ & $13.00[12.00,14.00]$ & $19.00[17.00,22.00]$ & $<0.001$ \\
\hline & Bicarbonate (mEq/L) & $24.00[22.00,25.00]$ & $23.00[20.75,24.00]$ & $18.00[16.00,21.75]$ & $<0.001$ \\
\hline & Creatinine $(\mathrm{mg} / \mathrm{dL})$ & $0.80[0.70,1.10]$ & $1.00[0.80,1.33]$ & $1.50[1.12,2.08]$ & $<0.001$ \\
\hline & Blood urea nitrogen (mg/dL) & $15.00[13.00,18.00]$ & $18.00[14.00,23.25]$ & $24.00[18.00,28.00]$ & $<0.001$ \\
\hline & Hematocrit (\%) & $24.60[21.10,27.00]$ & $25.00[21.48,29.00]$ & $25.60[21.00,27.60]$ & 0.613 \\
\hline & Hemoglobin (g/dL) & $8.40[7.50,9.65]$ & $8.50[7.30,9.90]$ & $8.50[7.10,9.57]$ & 0.69 \\
\hline & PTT (sec) & $40.70[35.95,50.80]$ & $41.25[33.45,50.05]$ & $62.05[39.00,130.43]$ & $<0.001$ \\
\hline & PT (sec) & $15.90[14.85,17.30]$ & $15.50[14.30,17.12]$ & $17.00[14.83,19.67]$ & 0.007 \\
\hline & INR & $1.50[1.30,1.70]$ & $1.40[1.30,1.70]$ & $1.80[1.33,2.30]$ & 0.001 \\
\hline & $\begin{array}{l}\text { white blood cell count } \\
(\mathrm{K} / \mu \mathrm{L})\end{array}$ & $12.70[9.90,15.30]$ & $12.60[10.20,16.00]$ & $12.00[9.43,16.18]$ & 0.817 \\
\hline & Platelet count $(\mathrm{K} / \mu \mathrm{L})$ & $124.00[97.00,151.50]$ & $125.00[89.00,157.00]$ & $93.50[58.25,141.75]$ & 0.001 \\
\hline \multicolumn{6}{|c|}{ Treatment } \\
\hline & Extracorporeal circulation & $120(79.5 \%)$ & $115(57.5 \%)$ & $15(27.8 \%)$ & $<0.001$ \\
\hline & Bypass surgery & $6(4.0 \%)$ & $7(3.5 \%)$ & $3(5.6 \%)$ & 0.789 \\
\hline & Ventilation in firstday & $147(97.4 \%)$ & $186(93.0 \%)$ & $49(90.7 \%)$ & 0.104 \\
\hline
\end{tabular}


Table 2 (continued)

\begin{tabular}{|c|c|c|c|c|}
\hline Variables & $\begin{array}{l}\text { Anion gap }<12 \mathrm{mEq} / \mathrm{L} \\
(\mathrm{N}=151)\end{array}$ & $\begin{array}{l}\text { Anion gap between } \\
12-16 \mathrm{mEq} / \mathrm{L} \\
(\mathrm{N}=200)\end{array}$ & $\begin{array}{l}\text { Anion gap }>16 \mathrm{~m} \mathrm{Eq} / \mathrm{L} \\
(\mathrm{N}=54)\end{array}$ & $P$ value \\
\hline Urine output in first day (ml) & $2205.00[1723.50,3217.00]$ & $1982.50[1268.50,3012.50]$ & $1006.50[489.50,1968.50]$ & $<0.001$ \\
\hline \multicolumn{5}{|l|}{ Severity scores } \\
\hline GCS & $15.00[14.00,15.00]$ & $15.00[14.75,15.00]$ & $15.00[15.00,15.00]$ & 0.115 \\
\hline SOFA & $5.00[3.00,7.00]$ & $5.00[4.00,8.00]$ & $8.00[5.25,10.00]$ & $<0.001$ \\
\hline SAPSII & $31.00[26.00,38.50]$ & $32.50[27.00,42.00]$ & $46.50[40.00,58.50]$ & $<0.001$ \\
\hline
\end{tabular}

The continuous data's normality was tested with Shapiro-Wilk normality test. And in two independent groups compare, continuous variables with normal distribution would be represented by mean with standard deviation (SD), compared with t-test. Continuous variables with abnormal distribution would be represented by median and interquartile range, compared with Wilcoxon rank sum test. Categorical variables would be represented by frequency and percentage, compared with chi-square test. Abbreviation: LOS, length of stay; PTT, partial thromboplastin time; PT, prothrombin time; INR, international normalized ratio; ICU, intensive care unit; GCS, Glasgow Coma Scale; SOFA, sequential organ failure assessment; SAPSII, simplified acute physiology score II

Table 3 Compare of risk discrimination of anion gap with simplified acute physiology score II

\begin{tabular}{|c|c|c|c|c|c|c|c|}
\hline \multirow[t]{2}{*}{ Outcome } & \multirow{2}{*}{$\begin{array}{l}\text { Continuous AG } \\
\text { AUC }(95 \% \mathrm{Cl})\end{array}$} & \multicolumn{6}{|l|}{ SAPSII } \\
\hline & & $\operatorname{AUC}(95 \% \mathrm{Cl})$ & $P$ value ${ }^{a}$ & NRI $(95 \% \mathrm{CI})$ & $P$ value ${ }^{b}$ & IDI (95\%) & $P$ value $^{c}$ \\
\hline In-ICU mortality & $0.882(0.818-0.946)$ & $0.839(0.771-0.908)$ & 0.215 & $0.302(-0.074-0.677)$ & 0.115 & $0.118(-0.013-0.249)$ & 0.077 \\
\hline In-Hospital mortality & $0.861(0.785-0.937)$ & $0.833(0.767-0.899)$ & 0.441 & $0.373(0.009-0.738)$ & 0.045 & $0.110(-0.011-0.232)$ & 0.076 \\
\hline 90-day mortality & $0.809(0.722-0.895)$ & $0.801(0.730-0.872)$ & 0.847 & $0.176(-0.158-0.511)$ & 0.302 & $0.082(-0.014-0.179)$ & 0.095 \\
\hline $\begin{array}{l}\text { 1-year } \\
\text { mortality }\end{array}$ & $0.775(0.700-0.850)$ & $0.780(0.714-0.845)$ & 0.878 & $0.304(0.007-0.601)$ & 0.045 & $0.095(0.022-0.167)$ & 0.01 \\
\hline
\end{tabular}

a. For Delong' test of two correlated ROC curves of AG and SAPSII

b. For NRI between AG model and SAPSII model

c. For IDI between AG model and SAPSII model

Abbreviation: AG, anion gap; SAPSII, simplified acute physiology score II; ICU, intensive care unit; AUC, area under the curve; NRI, net reclassification index; IDI, integrated discrimination improvement; ROC, receiver operating characteristic

$12 \mathrm{mEq} / \mathrm{L}$, but became gentler (still positive correction) in the RCS (Fig. 2B) which was adjusted for admission information and severity score, including admission type, age, gender, aortic rupture, SOFA, SAPSII and Glasgow coma scale (GCS).

\section{Subgroup analysis}

As is shown in Fig. 3, subgroup analysis was conducted in different groups and was shown as forest plot. AG was significantly associated with in-ICU mortality in total cohort ( $P$ value $<0.05)$, all groups except sepsis identified group ( $P$ value $=0.248$ ). The results of interaction had shown that only gender had interactions with AG in predicting in-ICU mortality $(P$ value $<0.05)$.

\section{Multivariate logistic regression}

As is shown in Table 4, the crude model and the 4 models adjusted by other variables (Model I: adjusted for admission information and severity score; Model II: adjusted for complication and operation; Model III: adjusted for laboratory indicators; Model IV: full variables model, adjusted for admission information, severity score, complication, operation and laboratory indicators) all showed statistical significance $(P$ value $<0.05)$.

\section{Clinical benefit estimation}

As is shown in Fig. 4, 4 DCAs showed the clinical usefulness of each model through drawing the curves which reflected the relationship between potential threshold probability of in-ICU mortality, in-hospital mortality, 90-day mortality and 1-year mortality respectively ( $x$ axis) and the net benefit of using the model to stratify the risk of patients ( $y$ axis). Through these DCAs, we could discover that in the range of risk of in-ICU mortality, inhospital mortality, 90-day mortality and 1-year mortality, model 4 always had more net benefits compared with other models and crude AG.

\section{Discussion}

Through multivariate analysis, we found that $1 \mathrm{mEq} / \mathrm{L}$ increase of AG improved up to $42.3 \%$ (95\% CI: $28.5 \%$ $59.8 \%)$ risk of in-ICU mortality of AA patients who received OSR, which was statistically significant $(P$ value $<0.001)$. 

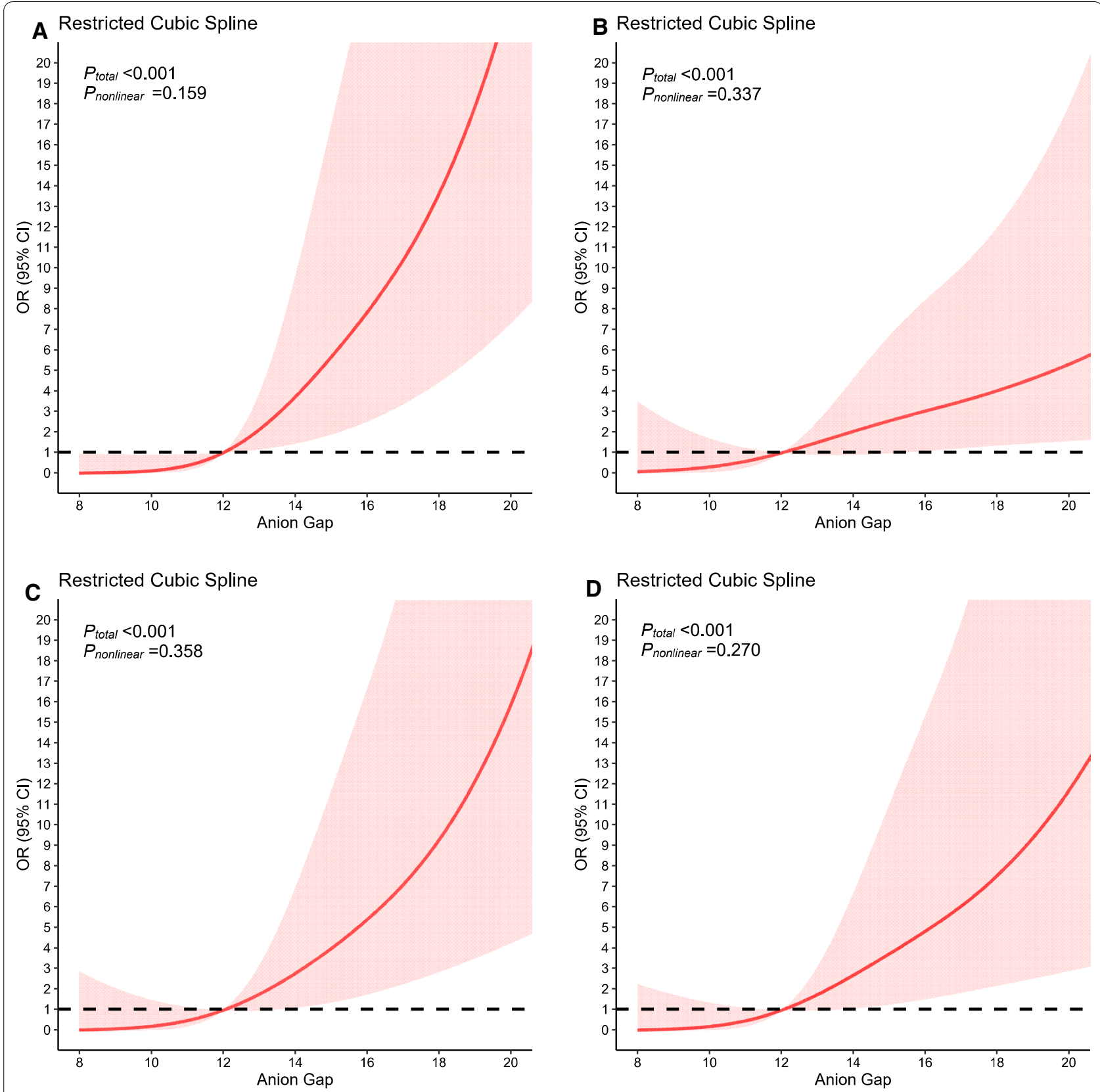

Fig. 2 Effects of anion gap on risk of mortality shown in restricted cubic splines. A Non-adjusted. B Adjusted for admission information and severity score, including admission type, age, gender, aortic rupture, SOFA, SAPSII and GCS; C Adjusted for complication and operation, including sepsis, chronic pulmonary diseases, peripheral vascular diseases, hypertension, renal failure, coagulopathy, fluid and electrolyte disorders, extracorporeal circulation, bypass surgery, ventilation on first day and urine output on first day; D Adjusted for laboratory indicators, including bicarbonate, creatinine, blood urea nitrogen, hematocrit, hemoglobin, PTT, PT, INR, white blood cell count and platelet count. In all figures, three-nodes restricted cubic splines were conducted to flexibly model and visualize the relation of anion gap (AG) with intensive care unit (ICU) mortality. $P_{\text {nonlinear }}>0.05$ in all models means that AG doesn't have statistically significant nonlinear relationship with ICU mortality. Abbreviation: OR, odds ratio; PTT, partial thromboplastin time; PT, prothrombin time; INR, international normalized ratio; GCS, Glasgow Coma Scale; SOFA, sequential organ failure assessment; SAPSII, simplified acute physiology score II

As we grouped patients by their admission level of AG, we found that death and LOS between those groups were all different. It proved that the subgroups divided by AG really indicated the different risk of death and hospital or ICU stay. Moreover, as we compared the risk discrimination ability of AG model and SAPSII, we found that there 


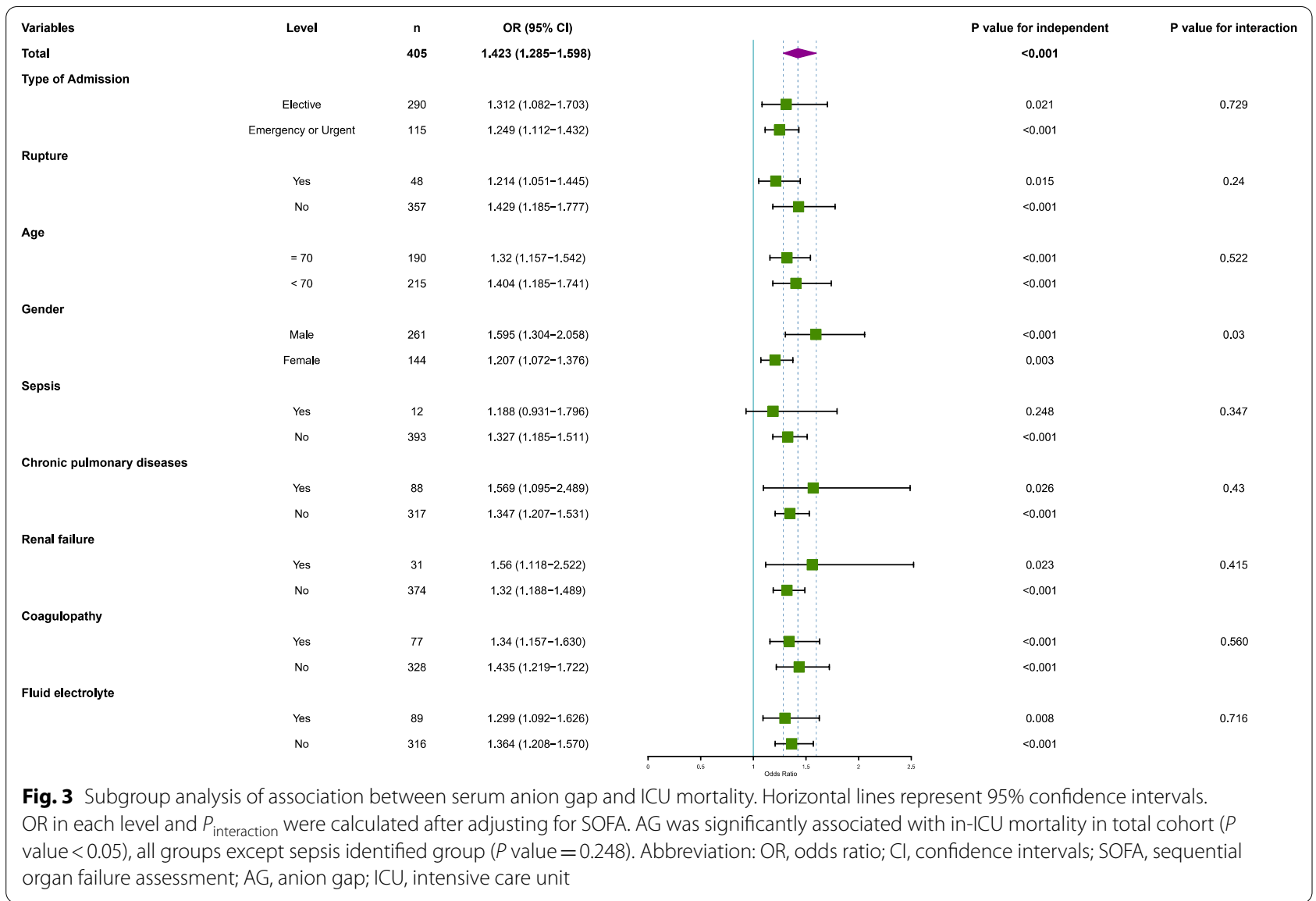

Table 4 Multivariate logistic regression for effects of anion gap on intensive care unit mortality

\begin{tabular}{llr}
\hline AG & OR $(\mathbf{9 5} \% \mathbf{C l})$ & $\boldsymbol{P}$ value \\
\hline Crude & $1.423(1.285-1.598)$ & $<0.001$ \\
Model I & $1.199(1.063-1.380)$ & 0.006 \\
Model II & $1.379(1.209-1.611)$ & $<0.001$ \\
Model III & $1.316(1.142-1.546)$ & $<0.001$ \\
Model IV & $1.286(1.053-1.651)$ & 0.025 \\
\hline
\end{tabular}

Crude, only includes anion gap in one model; Model I, adjusted for admission information and severity score, including admission type, age, gender, aortic rupture, SOFA, SAPSII and GCS; Model II, adjusted for complication and operation, including sepsis, chronic pulmonary diseases, peripheral vascular diseases, hypertension, renal failure, coagulopathy, fluid and electrolyte disorders, extracorporeal circulation, bypass surgery, ventilation on first day and urine output on first day; Model III, adjusted for laboratory indicators, including bicarbonate, creatinine, blood urea nitrogen, hematocrit, hemoglobin, PTT, PT, INR, white blood cell count and platelet count; Model IV, full variables model, adjusted for admission information, severity score, complication, operation and laboratory indicators above

Abbreviation: AG, anion gap; OR, odds ratio; LOS, length of stay; PTT, partial thromboplastin time; PT, prothrombin time; INR, international normalized ratio GCS, Glasgow Coma Scale; SOFA, sequential organ failure assessment; SAPSII, simplified acute physiology score II was no significant difference between the performance of these two models, except in hospital mortality and 1 year-mortality (AG model performed better). The AUC of AG for discrimination of survivors and non-survivor reached 0.882 (95\% CI 0.818-0.946), which was higher than our previous research that studied all kinds of AA patients in ICU (AUC: 0.8513, 95\% CI 0.7698-0.9328). Also, a report showed that EVAR caused smaller change of acid-base status than OSR [18]. These indicated that the prognostic prediction ability of AG might be better when dealing with patients who received OSR.

DCA and multivariate analysis both showed the good clinical usefulness and prediction ability of AG.

AG is a factor that indicates the acid-base status of patients. Traditionally, metabolic acidosis is categorized to the presence or absence of unmeasured anions, inferred by calculating AG [19]. Since it is reported that metabolic acidosis is a powerful marker of poor prognosis of critically ill patients, AG might be a prognostic factor for adverse clinical outcome in ICU patients [20].

In ruptured AAA, preoperative unmeasured AG is a prognosis factor for mortality, and elevated unmeasured AG is associated with lactic acid, ketoacids, uremia, and intoxications with nonchloride-containing acids as well 


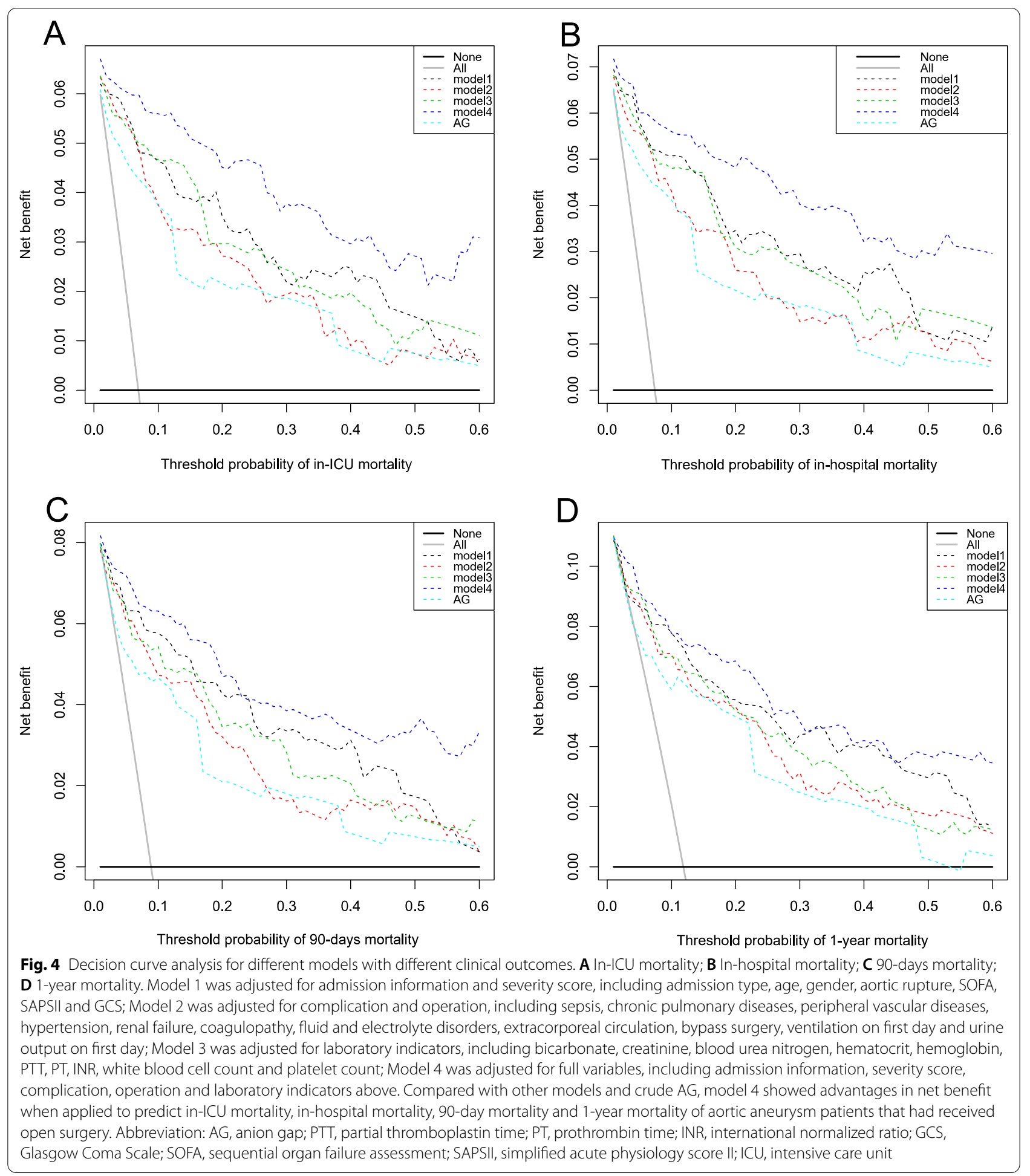

as in some forms of metabolic alkalosis [21]. In our study, an evidence of this hypothesis was that different levels of AG were significantly associated with different types of AA ( $P$ value $<0.001)$, as is shown in Table 2 .
Besides, several studies have indicated that aortic cross-clamp is associated with metabolic acidosis, which probably causes negative cardiovascular effects without proper intervention [18, 22]. Therefore, we inferred that an underlying preoperative acidosis, indicated by 
the elevated AG level on admission, might escalate when aortic cross-clamp is conducted, and eventually lead to a worse prognosis and a higher mortality when compared with the situation without preoperative acidosis. [18]

What's more, preoperative acidosis may be a surrogate for occult systemic malperfusion ostensibly, since lactate generated from hypoperfusion generates acidosis, and the resolution of lactic acidosis contributes to survival significantly $[23,24]$. In patients with acute type A aortic dissection, severe preoperative acidosis is associated with malperfusion or shock, and contributes to mortality $[25,26]$. A previous report demonstrated that acidosis included by lactate, pyruvate or $\mathrm{HCl}$ will increase whole blood viscosity and hematocrit; therefore, although systemic flow is normal or supranormal, increase of viscosity and hematocrit may partly contribute to regional hypoperfusion [24]. Lactic acidosis as a surrogate for systemic hypoperfusion or malperfusion is becoming an increasingly recognized and useful independent indicator of disease severity and a predictor of survival in the intensive care and trauma settings [23].

Also, preoperative acidosis may have associations with coagulopathy. It is indicated that acidosis is a probable reason for coagulopathy, and bleeding caused by coagulopathy contributes to intraoperative death of ruptured AAA patients [27]. Another study reported that in disseminated intravascular coagulation patients in the cardiothoracic surgical ICU, there is a strong relationship between acid-base derangement and mortality, since the elevated blood lactate concentration and base deficit is a reflection of severe tissue hypoxia and plays a key role in survival in critically ill patients [28]. In our study, as is shown in Table 2, different levels of AG are significantly associated with different incidences of coagulopathy ( $P$ value $<0.001$ ), which might be a supporting evidence of this hypothesis.

In addition to acid-base disturbance, it is reported that in geriatrics, the elevated AG level is a prognosis factor for mortality, since it is associated with hypertension, low cardiorespiratory fitness and decreased renal function [29]. Reports show that smoking, existence of other cardiovascular diseases, hypertension and dyslipidemia are risk factors of AAA, and untreated hypertension is also a risk factor for AAA rupture [1]. Besides, although smoking seems to have no significant relationship with TAA, hypertension is a risk factor for TAA, and hyperlipidemia is one of the determining factors of expansion rate of TAA, probably causing rupture [30-32]. These factors might cause negative effect on the prognosis of AA patients, and contribute to the elevated AG with the elevated mortality. The elevated incidence of cardiovascular events of AA patients is not associated to AA directly, but associated to the common risk factors and comorbidities.
In the current study, although chronic pulmonary diseases, hypertension and renal failure didn't show differences respectively between subgroups stratified by level of AG or those stratified by survival conditions, they might have a combined effect on AG and mortality, which probably explains the association between AG and mortality.

There are several limitations in this study. As this study is an observational study conducted by using an open database, we could just give a rough possible explanation of the mechanism that caused the association between AG level and mortality of AA patients in ICU after open surgery. Moreover, we did only obtain data from one database, for which we just conducted an internal validation in this study. An external validation is essential for proving the ability of our models in future studies. In addition, the net benefit of prediction models assessed by DCA was only showed through graphs, and there was not an exact numeric value that reflects the net benefit more precisely.

\section{Conclusions}

The level of serum AG is an important prognosis factor for AA mortality in ICU after open surgery, which might promote the refinement of the existing prediction models and the establishment of new models.

\begin{abstract}
Abbreviations
AA: Aortic aneurysm; AAA: Abdominal aortic aneurysm; AG: Anion gap; AUC : Area under curve; Cl: Confidence interval; CITI: Collaborative Institutional Training Initiative; DCA: Decision curve analysis; DREAM: Dutch Randomized Endovascular Aneurysm Management; EVAR: Endo-vascular aortic repair; GCS: Glasgow Coma Scale; ICD-9: International Classification of Diseases 9; ICD-9-CM: International Classification of Diseases, Clinical Modification; ICU: Intensive Care Unit; IDI: Integrated discrimination improvement; INR: International normalized ratio; LOS: Length Of Stay; MIMIC-III: Medical Information Mart for Intensive Care III; NRI: Net Reclassification Index; OSR: Open surgery repair; PT: Prothrombin time; PTT: Partial thromboplastin time; RCS: Restricted cubic spline; ROC: Receiver operating characteristic; SAPSII: Simplified Acute Physiology Score II; SD: Standard deviation; SOFA: Sequential Organ Failure Assessment; TAA: Thoracic aortic aneurysm; TEVAR: Thoracic endovascular aortic repair.
\end{abstract}

\section{Supplementary Information}

The online version contains supplementary material available at https://doi. org/10.1186/s12872-021-02263-4.

Additional file 1. Method - Data retrieval.

\section{Acknowledgements}

Not applicable.

\section{Authors' contributions}

KH, YJG, ZLH and RNS designed the research study. RNS collected the data and performed analysis. SRZ and GCY helped to perform programming. GCY, JC,XSG and SYP helped to write the manuscript. All authors read and approved the final manuscript. 


\section{Funding}

The project is sponsored by National Natural Science Foundation of China (Project Approval Number is 81800420). The funding body played no role in the design of the study and collection, analysis, decision to publish, interpretation of data and in writing the manuscript.

\section{Availability of data and materials}

The dataset supporting the conclusions of this article is freely available in the MIMIC-III database, which can be assessed on https://mimic.physionet.org. Researchers can formally request access to this free database by become a credentialed user on PhysioNet (https://physionet.org/) after completing the CITI (Collaborative Institutional Training Initiative) "Data or Specimens Only Research" course and approved by the institutional review boards of Beth Israel Deaconess Medical Center [11]. The datasets generated and/or analysed during the current study are not publicly available because we do not have access to data repositories open to the public at present, but are available from the corresponding author on reasonable request.

\section{Declarations}

\section{Ethics approval and consent to participate}

The authors are accountable for all aspects of the work in ensuring that questions related to the accuracy or integrity of any part of the work are appropriately investigated and resolved. This study was conducted in accordance with the Declaration of Helsinki (as revised in 2013). This study was approved by the institutional review boards of both Beth Israel Deaconess Medical Center and Massachusetts Institute of Technology Affiliates (authorization code: 35655780). Requirement for individual patient consent was waived because the project did not impact clinical care and all protected health information was deidentified, which was approved by the institutional review boards of both Beth Israel Deaconess Medical Center.

\section{Consent for publication}

Not applicable.

\section{Competing interests}

The authors declare that they have no competing interests.

\section{Author details}

'Department of Cardiovascular Surgery, Sun Yat-Sen Memorial Hospital, Sun Yat-Sen University, No. 33, Yingfeng Road, Haizhu District, Guangzhou 510000, Guangdong Province, China. ${ }^{2}$ Zhongshan School of Medicine, Sun YatSen University, No. 58, Zhongshan Rd. 2, Guangzhou 510080, Guangdong Province, China. ${ }^{3}$ Department of Cardiovascular Surgery, Guangzhou Red Cross Hospital, No. 396, Tongfu Middle Road, Guangzhou 510220, Guangdong Province, China.

Received: 16 May 2021 Accepted: 3 September 2021

Published online: 23 September 2021

\section{References}

1. Erbel R, et al. 2014 ESC Guidelines on the diagnosis and treatment of aortic diseases: Document covering acute and chronic aortic diseases of the thoracic and abdominal aorta of the adult. The Task Force for the Diagnosis and Treatment of Aortic Diseases of the European Society of Cardiology (ESC). Eur Heart J. 2014;35(41):2873-926.

2. Go AS, et al. Heart disease and stroke statistics-2013 update: a report from the American Heart Association. Circulation. 2013;127(1):e6-245.

3. Nordon IM, et al. Pathophysiology and epidemiology of abdominal aortic aneurysms. Nat Rev Cardiol. 2011;8(2):92-102.

4. Malecki C, et al. The role of inflammation and myeloperoxidase-related oxidative stress in the pathogenesis of genetically triggered thoracic aortic aneurysms. Int J Mol Sci. 2020;21(20):7678.

5. Elefteriades JA, Farkas EA. Thoracic aortic aneurysm clinically pertinent controversies and uncertainties. J Am Coll Cardiol. 2010;55(9):841-57.

6. Sakalihasan N, et al. Abdominal aortic aneurysms. Nat Rev Dis Primers. 2018;4(1):34.
7. Ameli-Renani S, Pavlidis V, Morgan RA. Secondary Endoleak Management Following TEVAR and EVAR. Cardiovasc Intervent Radiol. 2020:43(12):1839-54.

8. Thompson PC, et al. Predictive models for mortality after ruptured aortic aneurysm repair do not predict futility and are not useful for clinical decision making. J Vasc Surg. 2016;64(6):1617-22.

9. Kim MJ, et al. Serum anion gap at admission as a predictor of mortality in the pediatric intensive care unit. Sci Rep. 2017;7(1):1456.

10. Chen Q, et al. Serum anion gap on admission predicts intensive care unit mortality in patients with aortic aneurysm. Exp Ther Med. 2018;16(3):1766-77.

11. Johnson AE, et al. MIMIC-III, a freely accessible critical care database. Sci Data. 2016:3:160035.

12. Zhang Z. Multiple imputation with multivariate imputation by chained equation (MICE) package. Ann Transl Med. 2016;4(2):30.

13. Johnson $A E$, et al. The MIMIC Code Repository: enabling reproducibility in critical care research. J Am Med Inform Assoc. 2018;25(1):32-9.

14. Pollard TJ, Johnson AE, Blundell J. MIT-LCP/mimic-code: MIMIC-III v1.4. 2017 [cited 2020 08/08]; Available from: https://doi.org/10.5281/zenodo.821872

15. DeLong ER, DeLong DM, Clarke-Pearson DL. Comparing the areas under two or more correlated receiver operating characteristic curves: a nonparametric approach. Biometrics. 1988;44(3):837-45.

16. Pencina MJ, et al. Evaluating the added predictive ability of a new marker: from area under the ROC curve to reclassification and beyond. Stat Med. 2008;27(2):157-72.

17. Van Calster B, et al. Reporting and interpreting decision curve analysis: a guide for investigators. Eur Urol. 2018;74(6):796-804.

18. Thompson JP, et al. Cardiovascular and catecholamine responses during endovascular and conventional abdominal aortic aneurysm repair. Eur $J$ Vasc Endovasc Surg. 1999;17(4):326-33.

19. Al-Jaghbeer M, Kellum JA. Acid-base disturbances in intensive care patients: etiology, pathophysiology and treatment. Nephrol Dial Transplant. 2015;30(7):1104-11.

20. Gunnerson KJ. Clinical review: the meaning of acid-base abnormalities in the intensive care unit part I_epidemiology. Crit Care. 2005;9(5):508-16.

21. Shackleton $C R$, et al. Preoperative predictors of mortality risk in ruptured abdominal aortic aneurysm. J Vasc Surg. 1987;6(6):583-9.

22. Eide TO, et al. Changes in hemodynamics and acid-base balance during cross-clamping of the descending thoracic aorta. A study in patients operated on for thoracic and thoracoabdominal aortic aneurysm. Eur Surg Res. 2005;37(6):330-4

23. Bennett JM, et al. Hyperlactemia predicts surgical mortality in patients presenting with acute stanford Type-A aortic dissection. J Cardiothorac Vasc Anesth. 2017:31(1):54-60.

24. Kaplan LJ, Frangos S. Clinical review: acid-base abnormalities in the intensive care unit_-Part II. Crit Care. 2005;9(2):198-203.

25. Lawton JS, et al. The profound impact of combined severe acidosis and malperfusion on operative mortality in the surgical treatment of type A aortic dissection. J Thorac Cardiovasc Surg. 2018;155(3):897-904.

26. Ong CS, et al. The Strongest Risk Factor for Operative Mortality in Acute Type A Aortic Dissection is Acidosis: Validation of Risk Model. Semin Thorac Cardiovasc Surg. 2020;32(4):674-80

27. Fransson $M$, Rydningen $H$, Henriksson AE. Early coagulopathy in patients with ruptured abdominal aortic aneurysm. Clin Appl Thromb Hemost. 2012;18(1):96-9.

28. Demma $\sqcup$, et al. Predicting mortality in patients with disseminated intravascular coagulation after cardiopulmonary bypass surgery by utilizing two scoring systems. Blood Coagul Fibrinolysis. 2019;30(1):11-6.

29. Ahn SY, et al. Serum anion gap is predictive of mortality in an elderly population. Exp Gerontol. 2014:50:122-7.

30. Cheung $\mathrm{K}$, et al. Thoracic aortic aneurysm growth: role of sex and aneurysm etiology. J Am Heart Assoc. 2017;6(2):3792.

31. Sidloff $D$, et al. Mortality from thoracic aortic diseases and associations with cardiovascular risk factors. Circulation. 2014;130(25):2287-94.

32. Yiu RS, Cheng SW. Natural history and risk factors for rupture of thoracic aortic arch aneurysms. J Vasc Surg. 2016;63(5):1189-94.

\section{Publisher's Note}

Springer Nature remains neutral with regard to jurisdictional claims in published maps and institutional affiliations. 